\title{
O EXERCÍCIO DA REVISÃO E SEU TRATAMENTO EDITORIAL: PARA UMA EDIÇÃO DA POESIA DE PEDRO HOMEM DE MELLO
}

Elsa PEREIRA

FCT (SFRH/BPD/92155/2013) ${ }^{1}$; - Centro de Linguística da Universidade de Lisboa (CLUL)

\section{RESUMO}

Este artigo debruça-se sobre alguns poemas de Pedro Homem de Mello (1904-1984) que foram sujeitos a processos de reescrita profunda. Segundo a argumentação aqui exposta, por mais divergentes que possam ser, essas versões, resultantes de revisão vertical, não podem ser separadas e devem editar-se em simultâneo. Neste sentido, as abordagens digitais surgem como as mais adequadas, para cotejar, editar e reorganizar automaticamente os testemunhos, explorando a naturez̧a dinâmica da condição textual.

\section{ABSTRACT}

This article deals with a few poems by Pedro Homem de Mello (1904-1984), which were subject to rewriting through vertical revision. It argues that, no matter how different they may seem to be, versions cannot be unlinked from one another and should be brought together in a genetic-critical edition. In order to explore diachronic revision, electronic strategies must be devised, so that new patterns and relationships may be rearranged through automatic processing, thus exploring the dynamic textual condition.

\footnotetext{
${ }^{1}$ Este trabalho foi desenvolvido no âmbito do projeto de Pós-doutoramento "Poesia de Pedro Homem de Mello: edição crítico-genética", apoiado por uma bolsa de investigação da Fundação para a Ciência e a Tecnologia (SFRH/BPD/92155/2013), comparticipada pelo Fundo Social Europeu e por fundos nacionais do MCTES de Portugal.
}

(C) Revista da ABRALIN, v.16, n.1, p. 139-154, Jan./Fev./Mar./Abril de 2017. 


\section{PALAVRAS-CHAVE}

Edição crítico-genética. Digital. Revisão. Versões.

\section{KEYWORDS}

Digital. Genetic-critical edition. Revision. Versions.

\section{Introdução}

A apologia de um trabalho aturado de aperfeiçoamento dos textos, que se prolongue muito para lá do momento da composição, remonta, como sabemos, à Antiguidade Clássica. Em artigo recentemente publicado (PEREIRA, 2016), tivemos oportunidade de observar que o verbo rever aparecia já no Vocabulário Português \& Latino (1720), de Raphael Bluteau, com um sentido próximo da reescrita de um texto pelo seu autor, mas foi com o Modernismo que esta aceção se consolidou, coincidindo com uma importante mudança no paradigma editorial:

During the early twentieth century, most books went through the following stages: manuscript, typescript, galley proof, revised proof, page proof, first edition. [...] The important point about this multistage process is that it stimulated, rather than merely enabled, the act of revision (SULLIVAN, 2013: 38).

Segundo Hannah Sullivan, a pluralidade de suportes materiais a que se submetia a produção de um livro em inícios do século XX, aliada à maior abundância de papel e ao aparecimento da máquina de escrever, resultou numa multiplicação dos estádios redacionais e no prolongamento do processo compositivo, muitas vezes para lá do momento da publicação. Tal circunstância estimulou os escritores modernistas a empreenderem 
um tipo de revisão mais audaz do que o praticado até à altura. Em vez das habituais substituições vocabulares e sintáticas (com o propósito de refinar a expressão), foi-se generalizando um exercício de reescrita mais profundo, obtido por processos de excisão ou interpolação de sequências textuais, com o intuito de alterar a estrutura discursiva e o conteúdo intrínseco dos textos. Seguindo a célebre máxima de Ezra Pound make it new - esta modalidade revisória, que passou a exercer-se ao nível estrutural, está na origem de uma série de tendências de estilo ligadas ao experimentalismo dos textos (como a fragmentação, a justaposição, a expressão elíptica, a opacidade, a descontinuidade discursiva, etc.) e poderá relacionar-se com aquilo que G. Thomas Tanselle denomina de revisão vertical:

two types of revision must be distinguished: that which aims at altering the purpose, direction, or character of a work, thus attempting to make a different sort of work out of it; and that which aims at intensifying, refining, or improving the work as then conceived [...] thus altering the work in degree but not in kind. If one may think of a work in terms of a spacial metaphor, the first might be labelled "vertical revision," because it moves the work to a different plane, and the second "horizontal revision," because it involves alterations within the same plane (TANSELLE, 1990: 53).

Partindo de alguns exemplos na obra de um poeta do Segundo Modernismo português, este artigo procura refletir sobre as abordagens editoriais mais adequadas à natureza dinâmica das composições e a um conceito de textualidade flutuante, que se transforma e amplia, através de múltiplas manifestações. 


\section{Revisão vertical na poesia de P. Homem de Mello}

Pedro Homem de Mello (1904-1984) é um dos poetas proeminentes na lírica portuguesa do séc. XX, cuja extensa obra se encontra, em parte inédita (e dispersa por manuscritos ou dactiloscritos), em parte distribuída por uma variedade de publicações impressas (como periódicos, postais ilustrados e livros de poesia), bem como testemunhos áudio, onde se registam algumas adaptações musicais para o fado. ${ }^{2}$

Além da natureza multimédia que caracteriza esses registos (e, só por si, constituiria um desafio editorial), a obra do autor coloca-nos um problema particularmente estimulante em termos filológicos: o facto de os seus poemas terem sido sujeitos a processos de reescrita profunda, resultando frequentemente em versões díspares.

Em relação a estas, podemos distinguir um primeiro grupo em que o parentesco é mais ou menos evidente, já que o processo de revisão assenta em operações de excisão, extensão ou substituição de partes do poema, mantendo todavia intacto o cerne da composição. A título ilustrativo, confrontem-se, na fig. 1, dois dos oito testemunhos coligidos para o poema "Eternidade", que Pedro Homem de Mello publicou no jornal Soberania do Povo (A - MELLO, 1947, n. ${ }^{\circ}$ 5435; B - MELLO, 1947, n. ${ }^{\circ}$ 5436).

\footnotetext{
${ }^{2}$ Estas adaptações musicais foram já objeto de uma comunicação que apresentámos à 12 .t $^{\text {th }}$ International Conference of the European Society for Textual Scholarship (De Montfort University, Leicester, 19-21 novembro 2015): “When poems become songs: uses for an edition of Pedro Homem de Mello's poetry".
} 
FIGURA 1: Colação automática de dois testemunhos através da ferramenta Juxta.

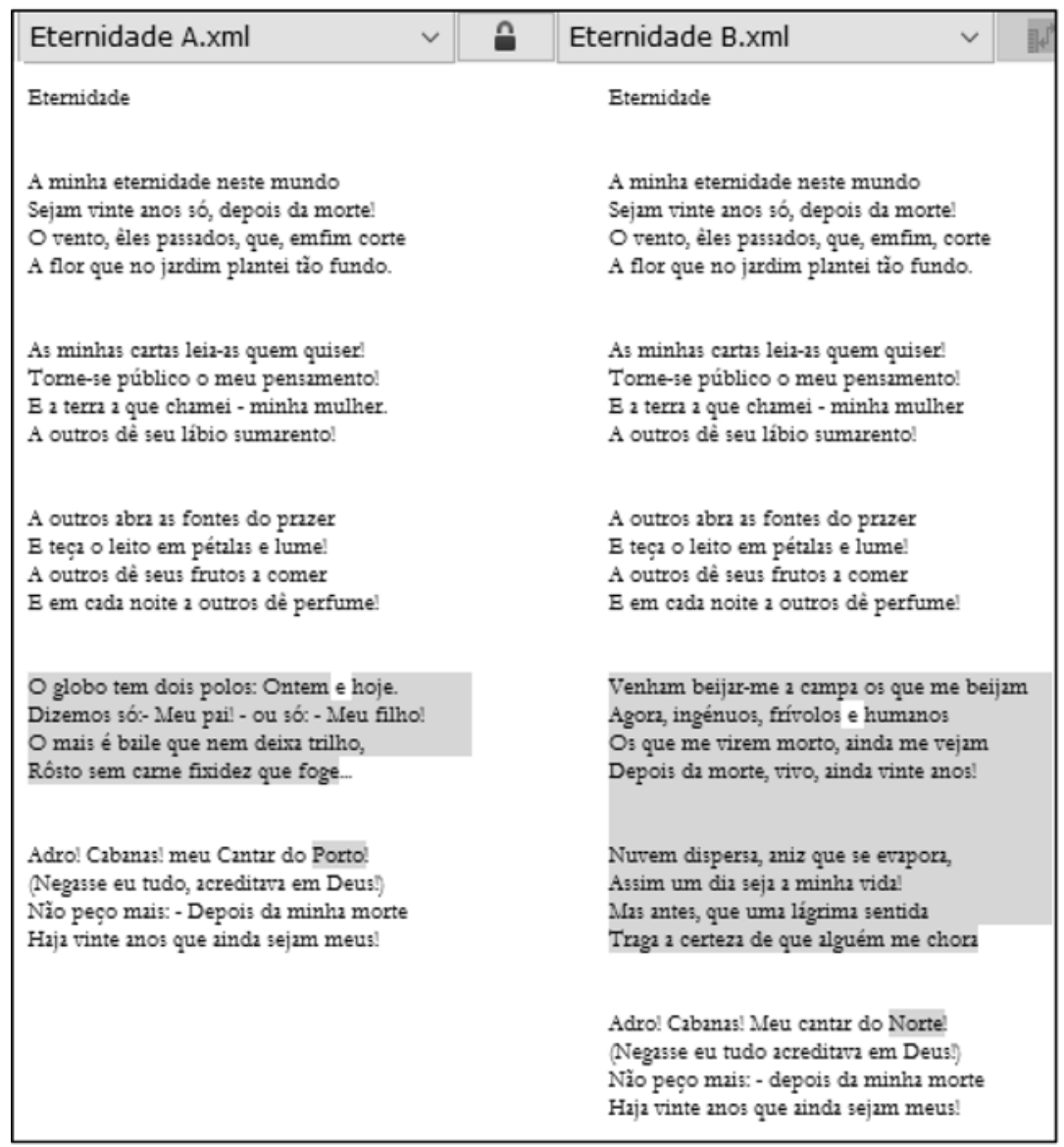

Casos há, no entanto, em que, entre as várias versões, parece existir uma relação mais de tipo filial, do que propriamente de descendência. Atente-se, neste sentido, ao cotejo entre "Saudade" (MELLO, 1961: 49) e "Apolo" (BNP, E14, cx. 22 [pasta 125]), ilustrado na fig. 2, onde é possível identificar alguns versos parcialmente comuns, que aliás aparecem dispostos em posição divergente. No exemplo em apreço, a dificuldade será tanto maior, se pensarmos que as transcrições 
apresentadas correspondem apenas a dois dos dezanove testemunhos até agora coligidos para um conjunto textual mais vasto, em que é possível identificar várias versões, com fenómenos extremos de variação entre si.

FIGURA 2: Colação automática de dois testemunhos através da ferramenta Juxta.

\begin{tabular}{|c|c|c|c|}
\hline Saudade.xml & $\checkmark$ & a & Apolo.xml \\
\hline $\begin{array}{l}\text { SAUDADE } \\
\text { Por onde andais ó ruas sinuosas } \\
\text { Da minha intância leve, embora triste? } \\
\text { Muros de pedra, a desfazer-se em rosas, } \\
\text { Diziam (mais que o altar!) que Deus existe. } \\
\text { Que é do Hermes de Praxiteles, achado } \\
\text { Sob os andrajos falsos de mendigo, } \\
\text { Chorando, ingénuo e tímido, a meu lado, } \\
\text { Quando a beleza e a paz iam consigo? } \\
\text { À beira-mar, sem música estrangeira, } \\
\text { A religião era o pecado e o Rei. } \\
\text { Azul, azul e branca era a bandeira. } \\
\text { Morri com ela. Quando foi? Nem sei... } \\
\text { Agora, o Sol parou e a luz destia } \\
\text { A sombra onde os sarcófagos estão. } \\
\text { Basta, por Deus! Dai-me, antes, a enxovia, } \\
\text { Mas devolvei-me toda a poesia } \\
\text { Da minha sexta-feira de pairão! }\end{array}$ & & & $\begin{array}{l}\text { APOLO } \\
\text { Hermes de Praxíteles, achado } \\
\text { Com trémulos andrajos de mendigo, } \\
\text { Tu que passavas, ontem, a meu lado, } \\
\text { Ignorando que o Sol fosse contigo! } \\
\text { Não sabias ainda, ail não sabias } \\
\text { Que o teu espelho era o cristal das águas } \\
\text { E que em teus lábios duas pionias } \\
\text { Vinham dar côr ao tédio, ao medo e às mágoas.. } \\
\text { Ail não sabias que existia o outeiro } \\
\text { E, por traz dele, intacto pinheiral, } \\
\text { Nem descobriras ainda o traiçoeiro } \\
\text { Perfil, no chão, da sombra do olivall } \\
\text { Se eras inútil, voluptuoso e belo } \\
\text { E de ti próprio nada, então, sabias, } \\
\text { Em que templo, em que impávido castelo } \\
\text { Fica a prega do manto que trazias? } \\
\text { Não! Não sabias que existia a lua } \\
\text { - A lua sobre os campo de centeio... } \\
\text { Mas veio o amor! E tu, que te escondias } \\
\text { Voltaste à Grécia, só por que ele veio! } \\
\text { Agora, o vento é para ti que é brisa } \\
\text { E o mar é para ti que lembra o rio } \\
\text { E nos teus olhos mágicos, desliza } \\
\text { A noite azul que envolve o meu navio... }\end{array}$ \\
\hline
\end{tabular}

Em situações deste tipo, poderíamos talvez perguntar qual o grau de variação necessário para que se instaure uma nova versão, e se duas versões tão díspares poderão ser encaradas como textos independentes:

Of the problems concerning the concept of versions $[\ldots]$, there are two that should be mentioned $[\ldots]$. The first is the problem of determining when the developing version has reached a degree of coalescence that can be identified as an essayed version. The second is the problem of determining if and when a second version 
has coalesced that should be considered as separate from the first. To discuss these problems we need several related concepts: time, content, function, and material. One should also note that concepts of intention and authority are crucial to the idea of versions (SHILLINGSBURG, 2000: 69-70).

Para responder à primeira pergunta, Peter Shillingsburg identificou quatro fatores que nos podem ajudar a aferir o grau de variação textual (i.e. tempo, conteúdo, função e suporte material). A segunda questão, no entanto, é mais sensível, e tem suscitado posições discordantes. Alguns críticos admitem que uma versão pode dar origem a novo texto, sempre que se quebre um certo grau de continuidade entre dois estádios redacionais (MCLAVERTY, 1991: 138). Outros consideram que, por muito diferentes que possam ser, duas versões nunca se tornam textos distintos, e em circunstância alguma poderão ser editadas separadamente (BRYANT, 2005: 85). John Bryant inclui-se no segundo grupo e recomenda que editemos várias versões em simultâneo (ibidem: 94), assinalando a variação entre diferentes testemunhos e conduzindo os leitores através desses itinerários textuais (ibidem: 123). Em vez de um aparato marginal, que remeta a informação para o rodapé ou para o final do volume, deverão conceber-se estratégias inclusivas, capazes de articular o texto crítico com o aparato, de modo a envolver o leitor, despertando-o para os chamados prazeres do texto fuído (ibidem: 133).

Se é certo que a recomendação de Bryant faz todo o sentido (sobretudo quando lidamos com versões autorais), a verdade, porém, é que tais objetivos são difíceis de alcançar em edição impressa. Mesmo que a ideia de um aparato integrado esteja subjacente a várias iniciativas em papel - desde as propostas de Hans Zeller, em inícios dos anos 70 (ZELLER, 1995: 41), até à edição de Hans Walter Gabler para o Ulysses (JOYCE, 1984) - poucos são os leitores capazes de decifrar um percurso 
de variação através de aparatos codificados, sobretudo quando as versões sejam formalmente muito diferentes. Em situações de variação estrutural que envolvam apenas duas versões, a alternativa mais viável poderá passar por uma edição sinóptica. No entanto, os constrangimentos materiais inerentes ao suporte impresso tornam-se especialmente flagrantes, se em causa estiverem múltiplas versões deste tipo. No caso acima mencionado, por exemplo, teríamos de editar os dezanove testemunhos em páginas consecutivas. Ficariam os leitores impossibilitados de alterar a ordem pela qual escolhêssemos apresentar as versões, ou sequer de empreender um cotejo simultâneo de todos os testemunhos - a não ser que recorrêssemos a um gigantesco desdobrável, semelhante ao proposto por Giuseppe Tavani (vd. BOCHICCHIO, 2008).

Pelo que fica assim exposto, somos levados a concluir que a semiótica particular do códice é demasiado restritiva, para editar textos sujeitos a fenómenos extremos de variação estrutural, como a observada em muitos autores modernistas e, concretamente, na poesia de Pedro Homem de Mello:

The principles outlined above can best be realized, perhaps only realized, through the extraordinary hypertextual features of the electronic medium (BRYANT, 2005: 145).

\section{Abordagens digitais}

Para ultrapassar os constrangimentos descritos e tirar partido da flexibilidade hipertextual ${ }^{3}$, têm surgido nos últimos anos vários projetos de edição eletrónica, sobretudo na Europa e nos Estados Unidos (FRANZINI et al., 2016: 171), ao ponto de alguns especialistas

\footnotetext{
3 "Hypertext, a term coined by Theodor H. Nelson in the 1960s, refers [...] to [...] text that branches and allows choices to the reader, best read at an interactive screen. As popularly conceived, this is a series of text chunks connected by links which offer the reader different pathways" (LANDOW, 1997: 3).
} 
admitirem que, num futuro próximo, a generalidade das edições críticas e genéticas deverá passar por este suporte:

We are $[. .$.$] probably only a few years away from the day$ when e-publication will become the primary format of choice for scholarly editions, with print on demand, in a more sophisticated form than it exists at present, serving as the secondary format for those parts of the edition required by the reader (EGGERT, 2009: 74).

Enquanto as primeiras iniciativas na área encaravam o meio digital como mera plataforma de publicação (não se afastando muito do conceito de e-book), a mais recente geração de edições eletrónicas representa já uma verdadeira mudança de paradigma:

Scholarly digital editions are scholarly editions that are guided by a digital paradigm in their theory, method and practice. [...] The scholarly edition undergoes a fundamental change that is triggered by the new possibilities of digital technologies of description, encoding and publication (SAHLE, 2016: 28, 37-38).

Trata-se, na verdade, de um tipo de edição radicalmente diferente, baseado numa lógica abstrata de codificação semântica ${ }^{4}$ e na recíproca integração do texto com o aparato, "reconstelando as relações textuais num espaço não coincidente com a ordem bibliográfica” (PORTELA, 2003). A vantagem mais evidente deste tipo de abordagem, no que diz respeito ao tratamento da variação estrutural, reside na possibilidade

\footnotetext{
${ }^{4}$ Notemos, por contraste, a ambiguidade dos símbolos usados em edições tradicionais, onde prepondera uma lógica visual, assente em formatações ou carateres especiais, e por isso também mais sujeita a perdas de informação, nomeadamente na passagem de processadores texto, como Microsoft Word, para programas de paginação, como Adobe InDesign (atualmente usado pelas editoras, na produção de livros).
} 
de compararmos várias versões em simultâneo, sem que nenhuma seja privilegiada hierarquicamente.

Para isso, é necessário que a sintaxe e as linguagens de marcação assumam um lugar de destaque na arquitetura editorial, nomeadamente através de esquemas e marcadores XML, como os que vêm sendo fixados pela Text Encoding Initiative (TEI). Este consórcio, fundado em 1988, tem como objetivo desenvolver uma norma internacional para a representação de textos em formato eletrónico ${ }^{5}$, que venha incrementar o seu processamento. Graças a uma implementação crescente (FRANZINI et al., 2016: 175), com o consequente reforço da interoperabilidade, tem sido possível assegurar, por um lado, o robustecimento das edições e, por outro, a partilha de programas e plataformas em projetos análogos.

Entre os vários recursos disponibilizados pela comunidade TEI, em aceso aberto, destacaremos apenas um, por responder, de forma geral, aos objetivos do nosso projeto e à especificidade dos desafios colocados pela variação estrutural da poesia melliana. Trata-se da Versioning Machine, ${ }^{6}$ uma interface gráfica concebida por Susan Schreibman, em 2000, que tem sido adotada em diversas edições, para lidar com fenómenos extremos de variação. A VM 5.0 foi disponibilizada no início de 2016 e tem como grande novidade a funcionalidade HTML5, que, no nosso caso, é particularmente útil para editar poemas adaptados ao fado, pois permite que ficheiros de som e imagem sejam incorporados e alinhados com o texto.

Através da arquitetura hipertextual e de um esquema conforme à norma TEI-P5, a Versioning Machine adequa-se essencialmente a uma abordagem crítico-genética, que privilegie o texto sobre o documento. Assim, as emendas do editor podem ser codificadas usando os elementos $<$ choice $>$, $<$ sic $>$ e $<$ corr $>$, enquanto a representação do processo de escrita fica a cargo dos fac-símiles dos manuscritos, do dispositivo

\footnotetext{
${ }^{5}$ Periodicamente revista e aumentada, a versão atual desta norma data de 2012 e designa-se TEI-P5: <http://www.tei-c.org/release/doc/tei-p5-doc.readme-2.8.0.html>.

${ }^{6}<$ v-machine.org $>$
} 
de paragénese (remetido para notas marginais) e das transcrições dos testemunhos. Estas aparecem depois apresentadas na interface gráfica, numa disposição paralela, como ilustra a fig. 3 .

FIGURA 3: Apresentação de três versões de "Trapézio" na Versioning Machine.

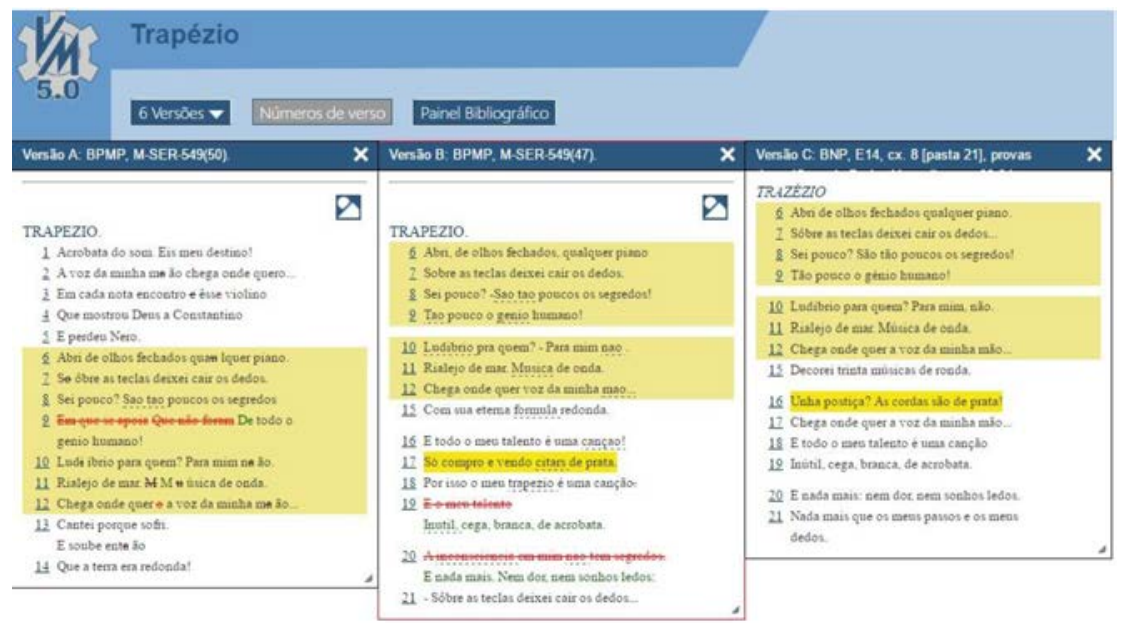

No exemplo apresentado, o poema intitula-se “Trapézio" e chegounos através de um conjunto de seis testemunhos, que começámos por codificar num único documento XML. A transformação para HTML foi depois executada, usando a opção "Apply Transformation Scenario" do oXygen ${ }^{7}$, de forma a articular na interface os vários painéis das versões, que podem ser selecionados e (re)ordenados pelo utilizador, em múltiplas combinações possíveis. Para facilitar a visualização, optámos por selecionar, na fig. 3, três versões, onde a variação estrutural é evidente: os cinco versos iniciais de $\mathrm{A}$ encontram-se omitidos em $\mathrm{B}$ e $\mathrm{C}$, enquanto os dois últimos versos de $\mathrm{A}$ aparecem substituídos em $\mathrm{B}$, por um bloco de sete versos, depois reescrito em $\mathrm{C}$.

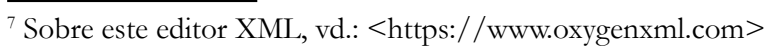


A interface da Versioning Machine permite que o utilizador interaja criticamente com a dinâmica da revisão, comparando aqueles blocos de texto, a par de outras marcações verso a verso ou palavra a palavra, que tenham sido codificadas pelo editor. Estas podem ocorrer em correspondência direta (e.g. versos $21 \mathrm{~B}$ e $21 \mathrm{C}$ ), ou entre diferentes versos de cada versão (e.g. versos B 17 e C 16).

Para que tal seja possível, é necessário que as transcrições dos testemunhos sejam sequenciadas e justapostas num aparato integrado, conforme prescrito pela norma TEI (que estabelece três métodos diferentes de codificar o aparato crítico: método de segmentação paralela, método de localização referenciada e método de dupla adjunção ${ }^{8}$ ). O esquema suportado pela Versioning Machine admite documentos codificados apenas com dois dos procedimentos prescritos pela Text Encoding Initiative, que poderão ser escolhidos pelo editor, em função da natureza do texto e dos problemas colocados. Enquanto o método de segmentação paralela constitui a abordagem mais simples e vantajosa (sendo por isso também o preferido pela generalidade dos editores ${ }^{9}$ ), nem sempre consegue lidar com a sobreposição de elementos e estruturas, que caracteriza o tipo de variação descrita neste artigo. Para ultrapassar o desafio, devemos, pois, recorrer ao método de localização referenciada, em que os vários versos correspondentes podem ser associados, através de um atributo $<$ loc $>$, adicionado aos respetivos aparatos:

FIGURA 4: Codificação dos versos C 16 e B 17 do poema “Trapézio”.

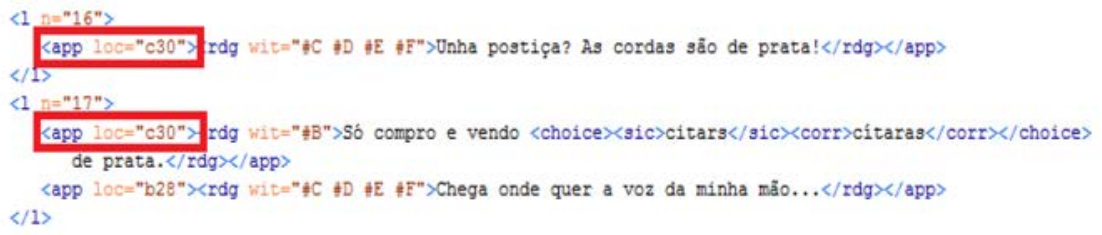

\footnotetext{
${ }^{8}$ Vd. "TEI Guidelines", capítulo 12 ("Critical Apparatus"), max. secção 12.2 ("Linking the Apparatus to the Text"): <http://www.tei-c.org/release/doc/tei-p5-doc/en/html/TC.html> ${ }^{9}$ Sobre a preferência do método de segmentação paralela sobre os restantes, vd.: < http://wiki. tei-c.org/index.php/Critical_Apparatus_Workgroup>
} 
Tal mecanismo fará que, ao passar o cursor sobre um dos versos associados com o mesmo valor de localização, o utilizador veja realçaremse, na interface gráfica, todas as variantes correlatas, independentemente da posição divergente que estas possam ocupar nas versões apresentadas.

Trata-se enfim de uma representação dinâmica da variação, significativamente distinta da que nos é facultada por abordagens estáticas e tradicionais. Lá onde as edições impressas privilegiam a fixação de um texto (modelo crítico) ou uma perspetiva teleológica (modelo genético), edições digitais deste tipo evidenciam uma conceção barthesiana de metamorfose textual, que é intrínseca às obras do período modernista, pois, como vimos, os seus autores reviam os textos mais frequentemente, mais estrutural e experimentalmente (SULLIVAN, 2013: 22). Daí que a decisão de editar a poesia de Pedro Homem de Mello através de uma abordagem heurística em suporte digital não seja apenas uma questão de forma ou de representação. Implica, mais que tudo, uma reconceptualização do próprio texto, entendido como entidade plural e fluída, que se transforma continuamente, assumindo várias manifestações:

In contrast to the one-text paradigm of the print edition, the digital edition shows a strong tendency towards multiple texts. Varying forms of text are not just teleologically moving toward one final goal. Rather, this conveys and embodies a pluralistic notion of text (SAHLE, 2016: 30-31).

\section{Conclusão}

Por tudo isto, concluiremos que uma abordagem digital, suportada pela codificação TEI, constitui talvez a solução mais adequada para editar a poesia de Pedro Homem de Mello - um autor que, em consonância 
com o seu tempo, submetia os textos a processos de revisão vertical, conferindo-lhes uma mobilidade intrínseca que coincide enfim com o conceito de hipertexto eletrónico:

o hipertexto traz uma resposta tecnológica à $[\ldots]$ reconcepção [pós-moderna] da textualidade (que passa por aspectos como o abandono da linearidade, o texto como rede, o texto aberto, a dispersão do texto, a questão da intertextualidade, o tema dos múltiplos começos e fins e o descentramento do texto) (FURTADO, 2010: 42).

\section{Referências}

BOCHICCHIO, Maria. A crítica genética e a estética da produção: o modelo Tavani. Revista da Faculdade de Letras: Línguas e Literaturas. [Porto: Faculdade de Letras da Universidade do Porto], 2006 [2008], II Série, vol. XXIII, p. 261-282.

BRYANT, John. The Fluid Text: a Theory of Revision and Editing for Book and Screen. 4. ${ }^{\mathrm{a}}$ ed. Ann Arbor: The University of Michigan Press, 2005.

EGGERT, Paul. The Book, the E-text and the 'Work-site'. In: DEEGAN, Marilyn; SUTHERLAND, Kathryn (ed.). Text Editing, Print and the Digital World. Farnham: Ashgate, 2009, p. 63-82.

FRANZINI, Greta; TERRAS, Melissa; MAHONY, Simon. A catalogue of digital editions. In: DRISCOLL, Matthew J.; PIERAZZO, Elena (org.). Digital Scholarly Editing: Theories and Practices. Cambridge: Open Book Publishers, 2016, p. 161-182. 
FURTADO, José Afonso. Hipertexto revisited. Letras de Hoje. Porto Alegre: Pontifícia Universidade Católica do Rio Grande do Sul, 2010, vol. 45, n. ${ }^{\circ}$ 2, p. 31-55. <http://revistaseletronicas.pucrs.br/ojs/index. $\mathrm{php} /$ fale/article/viewFile/7525/5395> [abril de 2016]

JOYCE, James. Ulysses: A Critical and Synoptic Edition (ed. Hans Walter Gabler, Wolfhard Steppe, Claus Melchior). New York: Garland, 1984.

LANDOW, George P. Hypertext 2.0: The Convergence of Contemporary Critical Theory and Technology. BaltimoreLondon: John Hopkins University Press, 1997.

MCLAVERTY, James - Issues of identity and utterance: an intentionalist response to "textual instability". In: COHEN, Philip (ed.). Devils and Angels: Textual Editing and Literary Theory. CharlottesvilleLondon: University Press of Virginia, 1991, p. 134-151.

MELLO, Pedro Homem de. Eternidade. Soberania do Povo [Águeda, s.n.], 1947, n. 5435, p. 4; n. 5436, p. 4.

Trapézio [provas tipográficas do livro Bodas Vermelhas, 1947, p. 93-94]. Biblioteca Nacional de Portugal. E14, cx. 8 [pasta 21].

. Expulsos do Governo da Cidade. Porto: Livraria Galaica, 1961.

. Apolo [dactiloscrito]. Biblioteca Nacional de Portugal. E14, cx. 22 [pasta 125].

- Trapézio [dactiloscrito]. Biblioteca Pública Municipal do Porto. M-SER-549(50).

- Trapézio [dactiloscrito]. Biblioteca Pública Municipal do Porto. M-SER-549(47). 
PEREIRA, Elsa. Da revisão autoral na poesia de Valter Hugo Mãe: as edições de três minutos antes de a maré encher. In: NOGUEIRA, Carlos (org.). Nenhuma Palavra É Exata: Estudos sobre a Obra de Valter Hugo Mãe. Porto: Porto Editora, 2016, p. 60-74.

PORTELA, Manuel. Hipertexto como Metalivro, 2003. < http:// www1.ci.uc.pt/pessoal/mportela/arslonga/MPENSAIOS/hipertexto_ como_metalivro.htm $>$ [abril de 2016]

SAHLE, Patrick. What is a Scholarly Digital Edition? In: DRISCOLL, Matthew J.; PIERAZZO, Elena (org.). Digital Scholarly Editing: Theories and Practices. Cambridge: Open Book Publishers, 2016, p. 30-31.

SHILlingSBURG, Peter L. Resisting Texts: Authority and Submission in Constructions of Meaning. 4. ${ }^{\mathrm{a}}$ ed. Ann Arbor: University of Michigan Press, 2000.

SULLIVAN, Hannah. The Work of Revision. CambridgeMassachusetts: Harvard University Press, 2013.

TANSELlE, G. Thomas. Textual Criticism and Editing. Charlottesville: University Press of Virginia, 1990.

ZELLER, Hans. Record and interpretation: analysis and documentation as goal and method of editing. In: GABLER, Hans Walter; BORNSTEIN, George; PIERCE, Gillian Borland (ed.). Contemporary German Editorial Theory. Ann Arbor: University of Michigan Press, 1995, p. 17-58. 